\title{
Preliminary Report on Input Cover Number as a Metric for Propositional Resolution Proofs ${ }^{\star}$
}

\author{
Allen Van Gelder \\ University of California, Santa Cruz CA 95060, USA, \\ WWW home page: http://www.cse.ucsc.edu/^avg
}

\begin{abstract}
Input Cover Number (denoted by $\kappa$ ) is introduced as a metric for difficulty of propositional resolution derivations. If $\mathcal{F}=\left\{C_{i}\right\}$ is the input $\mathrm{CNF}$ formula, and clauses are regarded as sets of literals, then the input cover number of a clause $D$ based on $F$, denoted by $\kappa(D, F)$, is defined as the minimum number of clauses $C_{i}$ needed to form a superset of (i.e., cover) $D$. The $\kappa$ for a derivation is the maximum $\kappa$ of any clause in the derivation. Input Cover Number provides a refinement of the clause-width metric analyzed by Ben-Sasson and Wigderson in the sense that it applies to families of formulas whose clause width grows with formula size, such as pigeon-hole formulas and the family GT $(n)$ introduced by Krishnamurthy (Acta Informatica 1985). Ben-Sasson and Wigderson (JACM 2001) showed that if formula $\mathcal{F}$ has the property that every resolution refutation of $\mathcal{F}$ contains a clause of width $W$, then certain lower bounds can be expressed in terms of $(W-$ width $(F))$ for the shortest tree-like refutation and for the shortest refutation. It is shown here that both bounds apply with $(W-$ width $(F))$ replaced by $\kappa$. For families of formulas whose clause width is bounded by a constant, the two metrics provide essentially the same bounds. Evidence is presented that $\kappa$ may be a sharper metric than clause width for distinguishing polynomial families from super-polynomial families. Ben-Sasson and Wigderson showed that pigeon-hole formulas $\operatorname{PHP}(m, n)$ formulas require clause-width to be $\Omega(n)$, and it is well known that pigeon-hole formulas require refutation length to be exponential in $n$. Bonet and Galesi (Computational Complexity 2001) showed that $\mathrm{GT}(n)$ formulas also require clause-width to be $\Omega(n)$, although the GT $(n)$ family has polynomial-length refutations. It is shown here that $\kappa$ is $\Omega(n)$ for pigeon-hole formulas and is $O(1)$ for $\mathrm{GT}(n)$ formulas and variants of $\mathrm{GT}(n)$.
\end{abstract}

\section{Introduction}

The reader is assumed to be generally familiar with the propositional satisfiability problem, CNF formulas, and resolution derivations. Some definitions are briefly reviewed in Section 2, but are not comprehensive.

Ben-Sasson and Wigderson [3] showed that, if the minimum-length general resolution refutation for a CNF formula $\mathcal{F}$ has $S$ steps, and if the minimumlength tree-like refutation of $\mathcal{F}$ has $S_{T}$ steps, then there is a (possibly different)

\footnotetext{
* A short version of this paper appears in SAT06, Aug. 2006, Seattle
} 
refutation of $\mathcal{F}$ using clauses of width at most:

$$
\begin{aligned}
& w(\mathcal{F} \vdash \perp) \leq w(\mathcal{F})+c \sqrt{n \ln S} \\
& w(\mathcal{F} \vdash \perp) \leq w(\mathcal{F})+\lg S_{T} .
\end{aligned}
$$

Note that the $w(\mathcal{F})$ terms were omitted from their statement in the introduction, but appear in their statements of the theorems. The notation for this expression is:

- $n$ is the number of propositional variables in $\mathcal{F}$;

$-w(\mathcal{F})$ is the width of the widest clause in $\mathcal{F}$;

$-w(\mathcal{F} \vdash \perp)$ denotes the minimum resolution width of $\pi$ ranging over all resolution derivations that refute $\mathcal{F}$, where the resolution width of $\pi$, denoted $w_{\mathcal{F}}(\pi)$, is the width of the widest clause in $\pi$;

$-c$ is a constant, independent of $\mathcal{F}$;

$-\ln$ and $\lg$ denote natural and binary logs, respectively.

All formulas and clauses are propositional, clauses are disjunctions of literals, formulas are in CNF, unless specified otherwise.

Our first results essentially eliminate the $w(\mathcal{F})$ terms in the Ben-Sasson and Wigderson theorems [3], and replace resolution width by $\kappa_{\mathcal{F}}(\pi)$, the input cover number, as defined next, in Section 1.1. Input cover number is an improvement on the input distance metric proposed previously [10]. For families of formulas whose widest clause is bounded by a constant, input cover number and resolution width are essentially equivalent measures.

Our interest in input cover number stems from the indications that it separates polynomial families from super-polynomial families for a wide class of formulas that represent SAT encodings of constraint satisfaction problems. Typically, these problems have a small number of wide positive clauses stating that each element of a set must have a value in a certain finite domain, together with a large number of localized constraints on combinations of values among small numbers of elements.

Two prototypical and widely studied examples are the pigeon-hole family $\operatorname{PHP}(n+1, n)$ and the GT $(n)$ family. The latter family was introduced by $\mathrm{Kr}-$ ishnamurthy [7], who conjectured that it required super-polynomial resolution length. This conjecture remained open for more than a decade before Stålmarck demonstrated a polynomial-length solution. Both families have a similar appearance: $\Theta(n)$ clause width, $\Theta\left(n^{2}\right)$ propositional variables, $\Theta\left(n^{3}\right)$ clauses, and $\Theta\left(n^{3}\right)$ overall formula length. However, the pigeon-hole family has minimum resolution length in $\Omega\left(2^{n}\right)[6,3]$, whereas the GT $(n)$ family has minimum resolution length in $O\left(n^{3}\right)[9,4]$. The clause-width metric does not distinguish between these two families: after the standard transformations into 3-CNF, giving $\operatorname{EPHP}(n+1, n)$ and $\operatorname{MGT}(n)$, they both have lower bounds for $w(\mathcal{F} \vdash \perp)$ in $\Omega(n)[3,4]$. The input distance metric [10] also does not distinguish between these two families. We show that the input-cover-number metric distinguishes sharply between them: $\kappa(\operatorname{PHP}(n+1, n) \vdash \perp)$ is in $\Theta(n)$, whereas $\kappa(\mathrm{GT}(n) \vdash \perp)$ is in $\Theta(1)$. 


\subsection{Input Cover Number}

We define input cover number for nontautologous clauses (primarily derived clauses in a resolution proof) for input CNF formula $\mathcal{F}$.

Definition 1.1. (input cover number) All clauses mentioned are nontautologous. Let $D$ be a clause; let $C$ be an input clause, i.e., a clause of formula $\mathcal{F}$. Regard clauses as sets of literals. The input cover number of $D$ w.r.t. $\mathcal{F}$, denoted $\kappa_{\mathcal{F}}(D)$, is the minimum number of clauses $C_{i} \in \mathcal{F}$ such that $D \subseteq \bigcup_{i} C_{i}$, i.e., the cardinality of the minimum set cover.

For a resolution proof $\pi$ the input cover number of $\pi$ w.r.t. $\mathcal{F}$, denoted $\kappa_{\mathcal{F}}(\pi)$, is the maximum over $D \in \pi$ of the input cover numbers of $D$ w.r.t. $\mathcal{F}$.

When $\mathcal{F}$ is understood from the context, $\kappa(D)$ and $\kappa(\pi)$ are written. Following Ben-Sasson and Wigderson [3], $\kappa(\mathcal{F} \vdash D)$ denotes the minimum of $\kappa_{\mathcal{F}}(\pi)$ over all $\pi$ that are derivations of $D$ from $\mathcal{F}$.

\subsection{Summary of Results}

The theorems shown here are that, if $\pi$ is a resolution refutation of $\mathcal{F}$ and $\pi$ uses all clauses of $\mathcal{F}$ and the length of $\pi$ is $S$, then there is a refutation of $\mathcal{F}$ using clauses that have input cover number w.r.t. $\mathcal{F}$ that is at most:

$$
\begin{aligned}
& \kappa(\mathcal{F} \vdash \perp) \leq c \sqrt{n \ln S} ; \\
& \kappa(\mathcal{F} \vdash \perp) \leq \lg S_{T} .
\end{aligned}
$$

Also, we show that the pigeon-hole family of formulas $\operatorname{PHP}(m, n)$ require refutations with input cover number $\Omega(n)$, although they contain clauses of width $n$. This result suggests that input cover number provides a refinement of the clause-width metric as a measure of resolution difficulty. That is, when a family of formulas with increasing clause-width, such as $\operatorname{PHP}(m, n)$, is transformed into a bounded-width family, such as $\operatorname{EPHP}(m, n)$, and the bounded-width family has large resolution width, this is not simply because they rederive the wide clauses of the original family, then proceed to refute the original family. Rather, it is the case that wide clauses substantially different from those in the original family must be derived.

Although the results are promising in some cases, the input-cover-number metric has an inherent fragility. Although $\kappa(\mathrm{GT}(n) \vdash \perp)$ is in $\Theta(1)$ for the natural encoding of $\operatorname{GT}(n)$, for 3-CNF variant, $\kappa(\operatorname{MGT}(n) \vdash \perp)$ is necessarily the same order of magnitude as the clause-width lower bound, $w(\operatorname{MGT}(n) \vdash \perp)$, i.e., in $\Omega(n)$.

Also, the current paper does not have an independent proof, based on input cover numbers, that the pigeon-hole family in its natural encoding has an $\Omega\left(2^{n}\right)$ lower bound on refutation length. Recall that Bonet and Galesi showed that $w(\operatorname{MGT}(n) \vdash \perp)$ is in $\Omega(n)$, yet $\operatorname{MGT}(n)$ has a refutation in $\Theta\left(n^{3}\right)$ [4]. Due to the fragility of $\kappa$ mentioned in the previous paragraph, the following attractive conjecture must fail: If a family has $\kappa$ in $\Omega(n)$, its refutation length must be 
super-polynomial in $n$. Future work should address whether a more restricted form of this conjecture can be proven, which will apply to the pigeon-hole family in its natural encoding.

Another attractive conjecture that must fail is the following: If a family has $\kappa$ in $\Omega(1)$, its refutation length must be polynomial. Choose any hard family and add two clauses, one consisting of all the positive literals and one consisting of all the negative literals. (Of course, these clauses are subsumed by other clauses in the formula, but they are legal.) Any refutation now has 2 as its input cover number. Again, future work should examine more restricted forms of this conjecture, which eliminate trivial counter-examples like this. For example, eliminating unused clauses before computing $\kappa$ would rule out this specific trick, but more sophisticated tricks like this might survive.

Considering these fragilities, we conclude this summary with a meta-conjecture: Any "interesting" general theorems about bounds involving $\kappa$ will use a modified definition that is based on the underlying constraint satisfaction problems, rather than arbitrary propositional formulas. That is, literals in the CNF encoding will be linked to semantic elements of the constraint satisfaction problem and the metric will take these associations into account. For example, all positive literals in a clause that refer to the same CSP element and the same property, but assert various values for that property, might be considered to be "covered" by one constraint.

\section{Preliminaries}

\subsection{Notation}

This section collects notations and definitions used throughout the paper. Standard terminology for conjunctive normal form (CNF) formulas is used. Notations are summarized in Table 1. Although the general ideas of resolution and derivations are well known, there is no standard notation for many of the technical aspects, so it is necessary to specify our notation in detail.

Definition 2.1. (assignment, satisfaction, model) A partial assignment is a partial function from the set of variables into $\{$ false, true $\}$. This partial function is extended to literals, clauses, and formulas in the standard way. If the partial assignment is a total function, it is called a total assignment, or simply an assignment.

A clause or formula is satisfied by a partial assignment if it is mapped to true; A partial assignment that satisfies a formula is called a model of that formula.

A partial assignment is conventionally represented by the (necessarily consistent) set of unit clauses that are mapped into true by the partial assignment. Note that this representation is a very simple formula. 
Table 1. Summary of notations.

\begin{tabular}{|l|l|}
\hline$a, \ldots, z$ & Literal; i.e., propositional variable or negated propositional variable. \\
$\neg x$ & Complement of literal $x ; \neg \neg x$ is not distinguished from $x$. \\
$|x|$ & The propositional variable in literal $x ;$ i.e., $|a|=|\neg a|=a$. \\
$A, \ldots, Z$ & Disjunctive clause, or set of literals, depending on context. \\
$\mathcal{A}, \ldots, \mathcal{H}$ & CNF formula, or set of literals, depending on context. \\
$\pi$ & Resolution derivation DAG. \\
$\sigma$ & Total assignment, represented as the set of true literals.
\end{tabular}

\subsection{Resolution as a Total Function}

Defining resolution as a total function removes the need to include the weakening rule in the proof system. Numerous proof complexity papers include the weakening rule as a crutch to handle "life after restrictions" $[3,4,1]$. However, according to Alasdair Urquhart, the weakening rule might add power to some resolution strategies, such as linear resolution.

Definition 2.2. (resolution, subsumption, tautologous) A clause is tautologous if it contains complementary literals. All tautologous clauses are considered to be indistinguishable and are denoted by $T$.

If $C=[q, \alpha]$ and $D=[\neg q, \beta]$ are two non-tautologous clauses ( $\alpha$ and $\beta$ are subclauses), then

$$
\operatorname{res}(q, C, D)=\operatorname{res}(q, D, C)=\operatorname{res}(\neg q, C, D)=\operatorname{res}(\neg q, D, C)=[\alpha, \beta]
$$

defines the resolution operation, and $[\alpha, \beta]$ is called the resolvent, which may be tautologous. Resolution is extended to include $T$ as an identity element:

$$
\operatorname{res}(q, C, \top)=C
$$

provided $C$ contains $q$ or $\neg q$. 
Resolution is further extended to apply any two non-tautologous clauses and any literals, as follows. Fix a total order on the clauses definable with the $n$ propositional variables such that $\perp$ is smallest, $T$ is largest, and wider clauses are "bigger" than narrower clauses. Other details of the total order are not important.

If $C=[\alpha]$ does not contain $q$ and $D=[\neg q, \beta]$ is non-tautologous, then

$$
\operatorname{res}(q, C, D)=\operatorname{res}(q, D, C)=\operatorname{res}(\neg q, C, D)=\operatorname{res}(\neg q, D, C)=[\alpha]
$$

If $C=[\alpha]$ and $D=[\beta]$ and neither contains $q$ or $\neg q$, and both are nontautologous, then

$$
\begin{aligned}
\operatorname{res}(q, C, D) & =\operatorname{res}(q, D, C)=\operatorname{res}(\neg q, C, D)=\operatorname{res}(\neg q, D, C) \\
& =\text { the smaller of } C \text { and } D .
\end{aligned}
$$

With this generalized definition of resolution, we have an algebra, and the set of clauses (including $T$ ) is a lattice, based on $\subseteq$, with the convention that every clause is a subset of $T$. We shall see later that the benefit of this structure is that resolution "commutes up to subsumption" with restriction (see Definition 2.4), so restriction can be applied to any resolution derivation to produce another derivation.

If clause $C \subset D$, we say $C$ properly subsumes $D$; if $C \subseteq D$, we say $C$ subsumes $D$. Also, any non-tautologous clause properly subsumes $T$. Notation $D^{-}$is read as " $D$, or some clause that subsumes $D$ ".

Definition 2.3. (derivation, refutation) A derivation (short for propositional resolution derivation) from formula $\mathcal{F}$ is a rooted, directed acyclic graph (DAG) in which each vertex is labeled with a clause and possibly with a clashing literal. Let $D$ be the clause label of vertex $v$. If $D=C \in \mathcal{F}$, then $v$ has no out-edges and no clashing literal, and is called a leaf. Otherwise $v$ is called a resolution vertex, has two out-edges, say to vertices with clause labels $D_{1}$ and $D_{2}$, and is also labeled with the clashing literal $q$ such that

$$
D=\operatorname{res}\left(q, D_{1}, D_{2}\right),
$$

where res is the total function defined in Definition 2.2. In much of the discussion, vertices are referred to by their clause labels.

A derivation derives its root clause. When the root clause is $\perp$, the derivation is called a refutation.

\subsection{The Restriction Operation}

Definition 2.4. (restricted formula, restricted derivation) Let $\mathcal{A}$ be a partial assignment for formula $\mathcal{F}$. Let $\pi$ be a derivation from $\mathcal{F}$. The clause $C \mid \mathcal{A}$, read " $C$ restricted by $\mathcal{A}$ ", and the formula $\mathcal{F} \mid \mathcal{A}$, read " $\mathcal{F}$ restricted by $\mathcal{A}$ ", are defined as follows.

1. $C \mid \mathcal{A}=\top$, if $C$ contains any literal that occurs in $\mathcal{A}$. 
2. $C \mid \mathcal{A}=C-\{q \mid q \in C$ and $\neg q \in \mathcal{A}\}$, if $C$ does not contain any literal that occurs in $\mathcal{A}$. This may be the empty clause.

3. $\mathcal{F} \mid \mathcal{A}=\{C|\mathcal{A}| C \in \mathcal{F}\}$; i.e., apply restriction to each clause in $\mathcal{F}$.

Usually, occurrences of $T$ (produced by part (1)) are deleted in $\mathcal{F} \mid \mathcal{A}$.

4. $\pi \mid \mathcal{A}$ is the same DAG as $\pi$ structurally, but the clauses labeling the vertices are changed as follows. If a leaf (input clause) of $\pi$ contains $C$, then the corresponding leaf of $\pi \mid \mathcal{A}$ contains $C \mid \mathcal{A}$. Each derived clause of $\pi \mid \mathcal{A}$ uses resolution on the same clashing literal as the corresponding vertex of $\pi$.

The operation $\mathcal{F} \mid p$ (i.e., $\mathcal{F} \mid\{[p]\}$ ) is sometimes called "unit simplification".

The term "restrict" is sometimes called "strengthen" in the theorem-proving community [8]. Ben-Sasson and Wigderson [3] and others in the proof-complexity community use the term "restriction" for "unit simplification" or "restriction by a single literal"; several different terms for this operation may be found in the literature.

Example 2.5. Let $\mathcal{F}$ consist of clauses $C_{1}=[a, b], C_{2}=[\neg a, c], C_{3}=[\neg b, e]$, and $C_{4}=[\neg c, \neg d]$. Let $\pi$ consist of leaves $C_{1}, C_{2}$ and $C_{4}$ and the derived clauses

$$
\begin{aligned}
& D_{1}=\operatorname{res}\left(a, C_{1}, C_{2}\right)=[b, c], \\
& D_{2}=\operatorname{res}\left(c, D_{1}, C_{4}\right)=[b, \neg d], \\
& D_{3}=\operatorname{res}\left(b, D_{2}, C_{3}\right)=[e, \neg d] .
\end{aligned}
$$

Then $\mathcal{F} \mid a=\{[c],[\neg b, e],[\neg c, \neg d]\}$, Also, $\mathcal{F} \mid\{a, c\}=\{[\neg b, e],[\neg d]\}$.

Now consider $\pi \mid a$. The leaves are $C_{1}\left|a=\mathrm{T}, C_{2}\right| a=[c], C_{3} \mid a=C_{3}$, and $C_{4} \mid a=C_{4}$. The derived clauses are $E_{1}, E_{2}$ and $E_{3}$, where:

$$
\begin{aligned}
& E_{1}=\operatorname{res}(a, \top,[c])=[c] ; \\
& E_{2}=\operatorname{res}(c,[c],[\neg c, \neg d])=[\neg d] ; \\
& E_{3}=\operatorname{res}(b,[\neg d],[\neg b, e])=[\neg d] .
\end{aligned}
$$

Notice that $E_{i} \neq D_{i} \mid a$ in any case, but $E_{i}=\left(D_{i} \mid a\right)^{-}$in all cases. Also notice that the clashing literal is absent from one operand in the resolution for $E_{3}$, so the resolvent is just the other operand.

Lemma 2.6. Given formula $\mathcal{F}$, and a restriction literal $p$,

$$
\operatorname{res}\left(q, D_{1}\left|p, D_{2}\right| p\right) \subseteq \operatorname{res}\left(q, D_{1}, D_{2}\right) \mid p .
$$

Proof. The principal case that requires checking is when $q=p$ and $q \in D_{1}$ and $\neg q \in D_{2}$ (or vice versa). In this case,

$$
\operatorname{res}\left(q, D_{1}, D_{2}\right) \mid p=\operatorname{res}\left(q, D_{1}, D_{2}\right)=\left(D_{1}-p\right) \cup\left(D_{2}-\neg p\right) .
$$

Then $\operatorname{res}\left(q, D_{1}\left|p, D_{2}\right| p\right)=D_{2} \mid p=\left(D_{2}-\neg p\right)$. Therefore, $D_{2}\left|p \subseteq \operatorname{res}\left(q, D_{1}, D_{2}\right)\right| p$. 
Lemma 2.7. Given formula $\mathcal{F}$, and a restriction literal $p$, if $\pi$ is a derivation of $C$ from $\mathcal{F}$, then $\pi \mid p$ is a derivation of $(C \mid p)^{-}$(a clause that subsumes $\left.C \mid p\right)$ from $\mathcal{F} \mid p$.

Proof. The proof is by induction on the structure of $\pi$ with edge $v \rightarrow w$ interpreted to mean that $v$ is greater than $w$. Thus, the base cases are the vertices that are clauses in $\mathcal{F}$, called the leaves. By Lemma 2.6, if a vertex of $\pi$ contains the derived clause $C$, and the two adjacent operand vertices satisfy the lemma, then the corresponding vertex of $\pi \mid p$ contains $(C \mid p)^{-}$.

If $C$ is the root of $\pi$ and $C \mid p \neq \top$, then a $T$-free derivation of $(C \mid p)^{-}$can be constructed from $\pi \mid p$ by changing all resolution vertices that have exactly one $T$ operand to "copy" vertices that use the non- $T$ operand, then deleting all the $T$ vertices, then compressing out all the copy vertices. Finally, the resulting DAG might have multiple sources, so delete all vertices that cannot be reached from the original root, which now contains $(C \mid p)^{-}$. This procedure does not change the clause in any vertex of $\pi \mid p$.

Notice that Ben-Sasson and Wigderson [3] define $\pi \mid p$ differently, as clauseby-clause restriction of the originally derived clauses. As Example 2.5 showed, this definition does not necessarily produce a derivation; they do not discuss this issue. The definitions used herein $d o$ ensure that the restriction of a derivation is a derivation, without using weakening. The point of Lemma 2.7 is that the clauses derived from the restricted formula are at least as strong as the clauseby-clause restrictions of the originally derived clauses.

\subsection{Input Cover Number and Restriction}

A few properties of input cover number on clauses that result from restriction are stated.

Lemma 2.8. Let $C$ be a clause of $\mathcal{F}$ and let $\mathcal{A}$ be a partial assignment. If $C \mid \mathcal{A} \neq \top$ (i.e., $\mathcal{A}$ does not satisfy $C$ ), then $\kappa_{\mathcal{F}}(C \mid \mathcal{A})=1$.

Proof. $C \mid \mathcal{A} \subseteq C$.

Lemma 2.9. Let $D$ be a clause of $\mathcal{F}$, let $\mathcal{A}$ be a partial assignment, and let $\mathcal{G}=\mathcal{F} \mid \mathcal{A}$. If $D \mid \mathcal{A} \neq \top$ (i.e., $\mathcal{A}$ does not satisfy $D$ ), then $\kappa_{\mathcal{F}}(D) \leq \kappa_{\mathcal{G}}(D \mid \mathcal{A})+|\mathcal{A}|$.

Proof. $D \subseteq D \mid \mathcal{A} \cup \mathcal{A}$, and $\kappa_{\mathcal{F}}((D \mid \mathcal{A}) \cup \mathcal{A}) \leq \kappa_{\mathcal{F}}(D \mid \mathcal{A})+|\mathcal{A}|$. But $\kappa_{\mathcal{F}}(D \mid \mathcal{A}) \leq$ $\kappa_{\mathcal{G}}(D \mid \mathcal{A})$.

\section{Size vs. Input Cover Number Relationships}

Ben-Sasson and Wigderson [3] derived size-width relationships that they describe as a "direct translation of [CEI96] to resolution derivations." Their informal statement, "if $\mathcal{F}$ has a short resolution refutation then it has a refutation with a small width," applies only when $\mathcal{F}$ has no wide clauses. 
This section shows that by using input cover number rather than clause width, the restriction on the width of $\mathcal{F}$ can be removed. That is, the relationships are restricted by removing the additive term, width $(\mathcal{F})$.

The use of restriction for recursive construction of refutations with special properties originates with Anderson and Bledsoe [2], who used it as a uniform framework for showing completeness of various restrictions on resolution, including linear resolution, set-of-support strategy, positive resolution, and others. Clegg et al. [5] used it in connection with Groebner-basis refutations. Ben-Sasson and Wigderson [3] used it to construct resolution refutations of small width. Van Gelder used it to construct resolution refutations of small input distance. We use it here to construct resolution refutations of small input cover number, closely following Ben-Sasson and Wigderson.

Lemma 3.1. Given formula $\mathcal{F}$, and a restriction literal $p$, let $\mathcal{G}=\mathcal{F} \mid p$. If derivation $\pi_{1}$ derives clause $D$ from $\mathcal{G}$ with input cover number $\kappa_{\mathcal{G}}\left(\pi_{1}\right)=(d-1)$, then there is a derivation $\pi_{2}$ that derives $(D+\neg p)^{-}$from $\mathcal{F}$ with input cover number $\kappa_{\mathcal{F}}\left(\pi_{2}\right) \leq d$.

Proof. Since $\mathcal{G}$ contains neither $p$ nor $\neg p$, we can assume w.l.o.g. that no vertices of $\pi_{1}$ have $p$ or $\neg p$ as the clashing literal. Define $\pi_{2}$ to have the same DAG structure as $\pi_{1}$, and the same clashing literal at each vertex, but wherever a leaf of $\pi_{1}$ is labeled with $C \mid p$, label the corresponding leaf of $\pi_{2}$ with $C$. Each clause of $\mathcal{F}$ has at most one additional literal, $\neg p$, compared to the corresponding clause of $\mathcal{G}$, or else contains $p$. But no clauses of $\mathcal{F}$ containing $p$ are leaves of $\pi_{2}$. Complete the clause labeling of $\pi_{2}$ according to the definition of resolution. Clearly $\pi_{2}$ derives $(D+\neg p)^{-}$. For each clause $E$ in $\pi_{2}$, the corresponding clause in $\pi_{1}$ is $E \mid p$. By Lemma $2.9, \kappa_{\mathcal{F}}(E) \leq \kappa_{\mathcal{G}}(E \mid p)+1$. So $\kappa_{\mathcal{F}}\left(\pi_{2}\right) \leq d$.

Lemma 3.2. Given formula $\mathcal{F}$, and a restriction literal $p$, let $\mathcal{G}=\mathcal{F} \mid p$ and $\mathcal{H}=\mathcal{F} \mid \neg p$. If derivation $\pi_{1}$ derives $\perp$ from $\mathcal{G}$ with input cover number $\kappa_{\mathcal{G}}\left(\pi_{1}\right)=$ $d-1$, and derivation $\pi_{2}$ derives $\perp$ from $\mathcal{H}$ with input cover number $\kappa_{\mathcal{H}}\left(\pi_{2}\right)=d$, then there is a derivation $\pi_{3}$ that derives $\perp$ from $\mathcal{F}$ with input cover number $\kappa_{\mathcal{F}}\left(\pi_{3}\right) \leq d$.

Proof. Using Lemma 3.1, there is a derivation $\pi_{4}$ that derives $[\neg p]^{-}$from $\mathcal{G}$ with input cover number $\kappa_{\mathcal{F}}\left(\pi_{4}\right) \leq d$. If the root of $\pi_{4}$ is $\perp$, let $\pi_{3}=\pi_{4}$ and we are done. Otherwise, construct $\pi_{3}$ as follows:

1. Use $\pi_{4}$ as the initial part of $\pi_{3}$. This part of $\pi_{3}$ has input cover number at most $d$ w.r.t. $\mathcal{F}$.

2. Resolve every clause of $\mathcal{F}$ that contains $p$ with the root of $\pi_{4}$, which contains $[\neg p]$. Call this set of resolvents $\mathcal{F}_{1}$. All of these resolvents have input cover number 1 w.r.t. $\mathcal{F}$ (Lemma 2.8 ), so they do not contribute to $\kappa_{\mathcal{F}}\left(\pi_{3}\right)$; also, they and are in $\mathcal{H}$.

3. Let $\mathcal{F}_{2}$ consist of those clauses in $\mathcal{F}$ that contain neither $\neg p$ nor $p$. Note that $\mathcal{F}_{1}+\mathcal{F}_{2}=\mathcal{H}$. 
4. Complete the derivation $\pi_{3}$ according to the derivation $\pi_{2}$, using clauses from $\mathcal{F}_{1}$ and $\mathcal{F}_{2}$ in place of $\mathcal{H}$ at the leaves of $\pi_{2}$. Since $\kappa_{\mathcal{F}}(D) \leq \kappa_{\mathcal{H}}(D)$ for any clause $D$, this part of $\pi_{3}$ has input cover number at most $d$ w.r.t. $\mathcal{F}$.

Thus $\kappa_{\mathcal{F}}\left(\pi_{3}\right) \leq d$.

Theorem 3.3. Let $\mathcal{F}$ be an unsatisfiable formula on $n \geq 1$ variables and let $d \geq 0$ be an integer. Let $S_{T}$ be the size of the shortest tree-like refutation of $\mathcal{F}$. If $S_{T} \leq 2^{d}$, then $\mathcal{F}$ has a refutation $\pi$ with input cover number $\kappa_{\mathcal{F}}(\pi) \leq d$.

Proof. The proof is by induction on the pair $(n, d)$ with the component-wise partial order, and follows Ben-Sasson and Wigderson [3], except that it uses input cover number and Lemma 3.2 above. The bases cases are $d=0$ or $n=1$, and are immediate. For $d>0$ and $n>1$ assume the theorem holds for smaller pairs. Let $x$ be the clashing literal at the root of $\pi$, a shortest tree-like refutation of $\mathcal{F}$. The children of the root are themselves the roots of tree-like derivations of $x$ and $\neg x$; call them $\pi_{1}$ and $\pi_{0}$. Assume the size of $\pi_{1}$ is at most $2^{d-1}$. But $\pi_{1} \mid \neg x$ is a tree-like refutation of $\mathcal{G}=\mathcal{F} \mid \neg x$. By the inductive hypothesis, $\mathcal{G}$ has a refutation $\pi_{2}$ with input cover number $\kappa_{\mathcal{G}}\left(\pi_{2}\right) \leq d-1$. Also, $\mathcal{H}=\mathcal{F} \mid x$ has at most $n-1$ variables, so by the inductive hypothesis, $\mathcal{H}$ has a refutation with input cover number $\kappa_{\mathcal{H}}\left(\pi_{1}\right) \leq d$. By Lemma $3.2, \mathcal{F}$ has a refutation $\pi$ with input cover number $\kappa_{\mathcal{F}}(\pi) \leq d$.

Corollary 3.4. $S_{T}(\mathcal{F}) \geq 2^{\kappa(\mathcal{F} \vdash \perp)}$.

Theorem 3.5. Let $\mathcal{F}$ be an unsatisfiable formula on $n \geq 1$ variables and let $d \geq 0$ be an integer. Let $S(\mathcal{F})$ be the size of the shortest refutation of $\mathcal{F}$. If $S(\mathcal{F}) \leq e^{\left(d^{2} / 8 n\right)}$, then $\mathcal{F}$ has a refutation $\pi_{1}$ with $\kappa_{\mathcal{F}}\left(\pi_{1}\right) \leq d$.

Proof. The proof is by induction on the pair $(n, d)$ with the component-wise partial order, and follows Ben-Sasson and Wigderson [3], except that it uses input cover number and Lemma 3.2 above. Their local variable $d$ is renamed to $f$ here and denotes the input cover number that causes a clause to be classified as $f a t ; f=\lceil\sqrt{2 n \ln S(\mathcal{F})}\rceil$. For any derivation $\pi$, let $\pi^{*}$ be the set of clauses $D \in \pi$ with $\kappa_{\mathcal{F}}(D)>f$. Define $a=2 n /(2 n-f)$. The theorem follows from this claim:

Claim: For all $b \geq 0$ and $1 \leq m \leq n$, if formula $\mathcal{G}$ has $m$ variables and $\pi$ is a refutation of $\mathcal{G}$ and $\left|\pi^{*}\right|<a^{b}$, then $\kappa(\mathcal{G} \vdash \perp) \leq f+b$.

Setting $b=f$ and $\mathcal{G}=\mathcal{F}$, and using the identity $-\ln (1-f / 2 n)>f / 2 n$, ensures that $a^{b} \geq S(\mathcal{F})$, so ensures the hypothesis, $\left|\pi^{*}\right|<a^{b}$, is true. Setting $d=2 f$ proves the theorem.

The claim is proved by induction on on the pair $(m, b)$ with the componentwise partial order. The base cases are $b=0$ or $m=1$, for which the claim is immediate, as $\left|\pi^{*}\right|=0$. For $b>0$ and $m>1$, there is some literal $x$ that appears in at least $\left|\pi^{*}\right| f / 2 n$ clauses of $\pi^{*}$. Let $\pi \mid x$ be as defined in Definition 2.4. By Lemma 2.7 and the discussion following it, there is a $T$-free derivation $\pi_{1}$ with 
the same nontautologous clauses as $\pi \mid x$. Then $\left|\pi_{1}^{*}\right| \leq(1-f / 2 n)\left|\pi^{*}\right| \leq a^{b-1}$. But $\pi_{1}$ refutes $\mathcal{G} \mid x$, so by the inductive hypothesis, $\kappa(\mathcal{G} \mid x \vdash \perp) \leq f+b-1$. Let $\pi_{0}$ be the T-free version of $\pi \mid \neg x$, which refutes $\mathcal{G} \mid \neg x$. Since $\mathcal{G} \mid \neg x$ has fewer than $m$ variables and $\left|\pi_{0}^{*}\right| \leq a^{b}$, by the inductive hypothesis, $\kappa(\mathcal{G} \mid \neg x \vdash \perp) \leq f+b$. Applying Lemma 3.2 proves the claim.

Corollary 3.6. $S(\mathcal{F}) \geq e^{\left(\kappa(\mathcal{F} \vdash \perp)^{2} / 8 n\right)}$.

\section{Pigeon-Hole Formulas}

The well-known family of Pigeon-Hole formulas for $m$ pigeons and $n$ holes $(\operatorname{PHP}(m, n))$ is defined by these clauses:

$$
\begin{aligned}
C_{i} & =\left[x_{i, 1}, \ldots, x_{i, n}\right] \quad \text { for } 1 \leq i \leq m \\
B_{i j k} & =\left[\neg x_{i, k}, \neg x_{j, k}\right] \quad \text { for } 1 \leq i \leq m, 1 \leq j \leq m, 1 \leq k \leq n .
\end{aligned}
$$

For the standard version, $m=n+1$. We shall show that any refutation of $\operatorname{PHP}(m, n)$ with $m>n$ has input cover number $\Omega(n)$. An (already known) exponential lower bound for tree-like refutations follows by Corollary 3.4, but no useful lower bound for general refutations follows by Corollary 3.6, since the " $n$ " in that corollary is the number of variables, which is $n m$ in the notation of this section. The method follows Ben-Sasson and Wigderson [3], except that it uses input cover number and the original PHP clauses of width $n$.

Theorem 4.1. Any refutation of $\operatorname{PHP}(m, n)$ with $m>n$ has input cover number at least $n / 6$.

Proof. For $1 \leq i \leq m$, define

$$
\mathcal{A}_{i}=\left\{C_{i}, B_{i j k}, 1 \leq j \leq m, 1 \leq k \leq n\right\}
$$

which consists of all the constraints on pigeon $i$. Define $\mu(D)$, the complexity of a clause $D$, as the minimum number of $\mathcal{A}_{i}$ 's needed to logically imply $D$. Then $\mu(\perp)=n+1$ and $\mu(C)=1$ where $C$ is any input clause. Suppose $I$ is the index set for a minimum-cardinality set of $\mathcal{A}_{i}$ 's that imply $D$ and $n / 3 \leq|I|<2 n / 3$. That is,

$$
\left(\bigwedge_{i \in I} \mathcal{A}_{i}\right) \rightarrow D
$$

is a tautology. Such an $I$ must exist, because $\mu\left(\operatorname{res}\left(q, D_{1}, D_{2}\right)\right) \leq \mu\left(D_{1}\right)+\mu\left(D_{2}\right)$.

Equation (5) holds if and only if the following is unsatisfiable (note that $\neg(D)$ constitutes a set of unit clauses):

$$
\left(\bigwedge_{i \in I} \mathcal{A}_{i}\right) \wedge \neg(D)
$$


Let $P_{0}$ be the set of pigeons (first index of variables) that have negative literals in $D$; let $P_{1}$ be the set of pigeons that have positive literals in $D$. If $D$ has at least $n / 3$ negative literals, then its input cover number is at least $n / 6$; assume this is not the case. Therefore, $I-P_{0}$ is nonempty.

The plan of the proof is to show that, if $I-P_{0}$ is nonempty and $D$ has fewer than $n / 3$ negative literals, either there is an assignment that satisfies (6) or $P_{1}$ has at least $n / 3-1$ pigeons. Table 2 illustrates some of the notation.

Since $I-P_{0}$ is nonempty, let $p \in I-P_{0}$ and let $I^{*}=I-\{p\}$. Thus $p$ is some pigeon whose hole is not forced by $\neg(D)$. By the minimality of $I$ there is an assignment $\sigma$ that makes $\neg(D)$ true, makes $\mathcal{A}_{p}$ false and satisfies $\mathcal{A}_{i}$ for $i \in I^{*}$. W.l.o.g. let $\sigma$ be chosen to have as few positive literals as possible. Then $\sigma$ sets all $x_{i k}=0$ for $i$ not in $I \cup P_{0} \cup P_{1}$ and $1 \leq k \leq n$. Further, $\sigma$ sets all $x_{p k}=0$, $1 \leq k \leq n$, since none of these positive literals occur in $\neg(D)$. Choose a function $k(i)$ for $i \in I^{*}$ such that $x_{i, k(i)}=1$ in $\sigma$. Necessarily, $k\left(i_{1}\right) \neq k\left(i_{2}\right)$ for distinct $i_{1}, i_{2} \in I^{*}$. Let $K$ be the set of indexes in the range 1 through $n$ that are not in the range of $k(i) ;|K| \geq n / 3+2$. These are the holes that are available for pigeon $p$.

Recall that $\sigma$ sets $x_{p k}=0$ for all $k \in K$. Also, $\sigma$ sets $x_{i k}=1$ for $i \in I^{*}$ and $k \neq k(i)$ only if $x_{i k} \in \neg(D)$. If, for any $k \in K, x_{p k}$ can be flipped to 1 and $x_{i k}$ can be set to 0 for all $i \neq p$ without falsifying $\neg(D)$, that would create a satisfying assignment for (6). Therefore, for each $k \in K, \neg(D)$ contains $\neg x_{p k}$ or $x_{i k}$ for some $i \neq p$. Since $|K|>n / 3$ and we assumed $D$ has fewer than $n / 3$ negative literals, it must be the case that $\neg(D)$ contains $\neg x_{p k}$ for some $k \in K$.

Finally, we argue that since $\neg(D)$ contains $\neg x_{p k}$, for some $k \in K$, it must contain $\neg x_{i k}$ for all $i \in I^{*}$. Suppose this fails for some $i$. Then modify $\sigma$ by setting $x_{p, k(i)}=1, x_{p, k}=0, x_{i, k(i)}=0$, and $x_{i, k}=1$ (see Table 2). This produces a satisfying assignment for (6).

To summarize, if $\neg(D)$ contains $\neg x_{p k}$ for some $k \in K$, then $D$ contains positive literals for at least $n / 3-1$ different pigeons, i.e., $\left|P_{1}\right| \geq n / 3-1$. Since each positive clause refers to only one pigeon, at least $n / 3-1$ positive clauses are needed to "cover" $D$ in this case.

Table 2. Changing $\sigma$ to expose a faulty index set $I$, in proof of theorem.

\begin{tabular}{|c|c|c|c|c|c|c|c|c|c|c|c|}
\hline \multirow{3}{*}{\multicolumn{5}{|c|}{$\begin{array}{c}D=\left[\neg x_{11}, x_{32}, x_{52}\right] \\
\text { Original } \sigma\end{array}$}} & \multirow{3}{*}{\multicolumn{2}{|c|}{$\neg(D)=\left[x_{11}\right] \wedge\left[\neg x_{32}\right] \wedge[$}} & \multirow{2}{*}{\multicolumn{5}{|c|}{$\begin{array}{r}I=\{2,3,5\}, \quad I^{*}= \\
\text { Modified } \sigma\end{array}$}} \\
\hline & & & & & & & & & & & \\
\hline & & & & & & & \multirow{2}{*}{$\begin{array}{l}\text { pige- } \\
\text { ons }\end{array}$} & \multicolumn{4}{|c|}{ holes } \\
\hline $\begin{array}{r}\text { pige- } \\
\text { ons }\end{array}$ & 1 & 2 & 3 & 4 & $i$ & $k(i)$ & & 1 & 2 & 3 & 4 \\
\hline 1 & 1 & 0 & 0 & 0 & 2 & 3 & 1 & 1 & $\overline{0}$ & 0 & 0 \\
\hline 2 & 0 & 0 & 1 & 0 & 3 & 4 & 2 & 0 & 1 & $O$ & 0 \\
\hline 3 & 0 & 0 & 0 & 1 & & & 3 & 0 & 0 & 0 & 1 \\
\hline 4 & 0 & 0 & 0 & 0 & & $\{1,2\}$ & 4 & 0 & 0 & 0 & 0 \\
\hline$p=5$ & 0 & 0 & 0 & 0 & & & $p=5$ & 0 & $O$ & 1 & 0 \\
\hline 6 & 0 & 0 & 0 & 0 & & & 6 & 0 & 0 & 0 & 0 \\
\hline
\end{tabular}




\section{The GT(n) Family}

The $\operatorname{GT}(n)$ family was proposed by Krishnamurthy [7], who conjectured that refutations required exponential length. Stålmarck demonstrated the first polynomial solution, then Bonet and Galesi found another [9,4]. However, both of these solutions produce derived clauses of width about double that of the input and have input cover numbers of two. This section describes a refutation with input cover number 3 , which also has no derived clause wider than an input clause. This new refutation is about half as long as those previously published.

For the GT $(n)$ family, there is an underlying semantic interpretation that guides our understanding. We suppose there is a set $W$ whose elements are denoted $w_{i}, 1 \leq i \leq n$. The propositional variables of $\mathrm{GT}(n)$ correspond to possible directed edges between distinct elements of this set. A variable is true if the edge is present. To describe the $\operatorname{GT}(n)$ formulas, we introduce some clause names.

Definition 5.1. The clauses of $\operatorname{GT}(n)$ are named as follows for indexes indicated.

$$
\begin{array}{rlr}
C_{n}(j) & \equiv[\langle 1, j\rangle, \ldots,\langle j-1, j\rangle,\langle j+1, j\rangle, \ldots,\langle n, j\rangle] & 1 \leq j \leq n \\
B(i, j) & \equiv[\neg\langle i, j\rangle, \neg\langle j, i\rangle] \quad 1 \leq i<j \leq n & \\
A(i, j, k) & \equiv[\neg\langle i, j\rangle, \neg\langle j, k\rangle,\langle i, k\rangle] \quad 1 \leq i, j, k \leq n \text { and } i, j, k \text { distinct. }
\end{array}
$$

The $C_{n}(j)$ are called long clauses; the others are short clauses.

Note that $A(i, j, k)$ and $B(i, j)$ collectively enforce that the directed edge relation is a partial order, hence it is acyclic. Finally, $C_{n}(j)$ asserts that element $j$ is not a source.

We now describe a refutation with input cover number 3 , which also limits derived clause width to that of the input. The construction is recursive. The base case is GT(1), in which $C_{1}(1)=\perp$, so refutation is immediate. For $\operatorname{GT}(n)$, where $n>1$, the refutation begins by deriving $C_{n-1}(m)$ for $1 \leq m \leq n-1$. Then GT $(n-1)$ is refuted.

It remains to show how $C_{n-1}(m)$ is derived from $C_{n}(m), C_{n}(n)$, and some of the $B(i, j)$ and $A(i, j, k)$, where $1 \leq m \leq n-1$. This subderivation is presented in Figure 1.

To reduce $\operatorname{GT}(n)$ to $\operatorname{GT}(n-1)$ requires $n(n-1)$ resolutions, so the whole refutation is length $n\left(n^{2}-1\right) / 3$.

The only non-tree-like part of the refutation is the multiple use of $C_{n}(n)$. At the leaves, $C_{n}(n)$ is an input clause, but inside the recursion it is derived; e.g., the last line of the figure when $m=n-1$. It turns out that the number of steps for the tree-like version of this scheme is about $n 2^{n}$.

The attentive reader may have noticed that the only $A(i, j, k)$ used were those with $j>i$ and $j>k$. Thus $\mathrm{GT}(n)$ is not minimally unsatisfiable. 


\begin{tabular}{|c|c|c|c|c|}
\hline & \multicolumn{2}{|c|}{ derived clause } & second operand & cover \\
\hline 0 & $C_{n}(n)$ & & $\swarrow B(m, n)$ & $C_{n}(n)$ \\
\hline 1 & $C_{n}(n)$ & $\begin{array}{l}-[\langle m, n\rangle] \\
+[\neg\langle n, m\rangle]\end{array}$ & $A(1$, & $C_{n}(n), B(m, n)$ \\
\hline 2 & $C_{n}(n)$ & $\begin{array}{l}-[\langle 1, n\rangle,\langle m, n\rangle] \\
+[\langle 1, m\rangle, \neg\langle n, m\rangle]\end{array}$ & ) & $C_{n}(m), C_{n}(n), B(m, n)$ \\
\hline 3 & $C_{n}(n)$ & $\begin{array}{l}-[\langle 1, n\rangle,\langle 2, n\rangle,\langle m, n\rangle] \\
+[\langle 1, m\rangle,\langle 2, m\rangle, \neg\langle n, m\rangle]\end{array}$ & $\swarrow^{A(3, n, m)}$ & $C_{n}(m), C_{n}(n), B(m, n)$ \\
\hline$n-2$ & $C_{n-1}(m)$ & $+[\langle n-1, m\rangle, \neg\langle n, m\rangle]$ & $\swarrow^{A(n-1, n, m)}$ & $C_{n}(m), C_{n}(n), B(m, n)$ \\
\hline$n-1$ & $C_{n-1}(m)$ & $+[\neg\langle n, m\rangle]$ & $\swarrow^{C_{n}(m)}$ & $C_{n}(m), B(m, n)$ \\
\hline$n$ & $C_{n-1}(m)$ & & & $C_{n}(m)$ \\
\hline
\end{tabular}

Fig. 1. Subderivation for refutation of $\operatorname{GT}(n)$.

\section{Conclusion}

We proposed the input cover number metric $(\kappa)$ as a refinement of clause width and input distance for studying the complexity of resolution. For families with wide clauses, the trade-off between resolution refutation size and input cover number is sharper than the trade-off between resolution refutation size and clause width.

We showed that any refutation of $\operatorname{PHP}(m, n)$ requires $\kappa \geq n / 6$. Moreover, the proof showed that this input cover number can arise in two possible ways: by having $n / 6$ negative literals in a derived clause, or by having $n / 3-1$ positive literals that refer to distinct pigeons.

We showed that $\operatorname{GT}(n)$ [7], which has general refutations of polynomial size [9], but for which tree-like refutations are exponential [4], behaves quite differently: its $\kappa=3$. This family can be modified so that regular refutations are also exponential [1]. The modified family also has $\kappa=3$. These results suggest (very tentatively) that $\kappa$ might be the sharper metric for general resolution, while clause-width is sharper for tree-like resolution.

Some open problems remain. Can input cover number improve the lower bound for weak $\operatorname{PHP}(m, n)$, where $m \gg n$ ? Ben-Sasson and Wigderson [3] transformed this problem into a family with clause width proportional to $\log m$. Are there other natural families to which input cover number can be applied? Is there a trade-off between regular refutation size and input cover number? Can input cover number be defined in terms of an underlying constraint satisfaction problem to avoid losing all power under a transformation into 3-CNF? 


\section{References}

1. Alekhnovich, M., Johannsen, J., Pitassi, T., Urquhart, A.: An exponential separation between regular and unrestricted resolution. In: Proc. 34th ACM Symposium on Theory of Computing. (2002) 448-456

2. Anderson, R., Bledsoe, W.W.: A linear format for resolution with merging and a new technique for establishing completeness. Journal of the ACM 17 (1970) $525-534$

3. Ben-Sasson, E., Wigderson, A.: Short proofs are narrow - resolution made simple. JACM 48 (2001) 149-168

4. Bonet, M., Galesi, N.: Optimality of size-width tradeoffs for resolution. Computational Complexity 10 (2001) 261-276

5. Clegg, M., Edmonds, J., Impagliazzo, R.: Using the Groebner basis algorithm to find proofs of unsatisfiability. In: Proc. 28th ACM Symposium on Theory of Computing. (1996) 174-183

6. Haken, A.: The intractability of resolution. Theoretical Computer Science 39 (1985) 297-308

7. Krishnamurthy, B.: Short proofs for tricky formulas. Acta Informatica 22 (1985) 253-274

8. Letz, R., Mayr, K., Goller, C.: Controlled integration of the cut rule into connection tableau calculi. Journal of Automated Reasoning 13 (1994) 297-337

9. Stålmarck, G.: Short resolution proofs for a sequence of tricky formulas. Acta Informatica 33 (1996) 277-280

10. Van Gelder, A.: Lower bounds for propositional resolution proof length based on input distance. In: Eighth International Conference on Theory and Applications of Satisfiability Testing, St. Andrews, Scotland (2005) 